\title{
PET Response Criteria in Solid Tumors Predicts Progression- Free Survival and Time to Local or Distant Progression After Chemotherapy with Regional Hyperthermia for Soft-Tissue Sarcoma
}

\author{
Wolfgang P. Fendler ${ }^{1}$, Mona Lehmann ${ }^{1}$, Andrei Todica ${ }^{1}$, Ken Herrmann ${ }^{2}$, Thomas Knösel ${ }^{3}$, Martin K. Angele ${ }^{4-6}$, \\ Hans Roland Dürr ${ }^{5-7}$, Josefine Rauch ${ }^{5,6,8}$, Peter Bartenstein ${ }^{1,5}$, Clemens C. Cyran ${ }^{9}$, Marcus Hacker ${ }^{10}$, and \\ Lars H. Lindner ${ }^{5,6,11}$ \\ ${ }^{I}$ Department of Nuclear Medicine, Ludwig-Maximilians-University of Munich, Munich, Germany; ${ }^{2}$ Department of Nuclear Medicine, \\ Julius-Maximilians-University of Würzburg, Würzburg, Germany; ${ }^{3}$ Institute of Pathology, Ludwig-Maximilians-University of Munich, \\ Munich, Germany; ${ }^{4}$ Department of General, Visceral, Transplantation, Vascular, and Thoracic Surgery, Ludwig-Maximilians-University \\ of Munich, Munich, Germany; ${ }^{5}$ Comprehensive Cancer Center, Ludwig-Maximilians-University of Munich, Munich, Germany; ${ }^{6}$ Center \\ for Bone and Soft Tissue Sarcoma (SarKUM), Ludwig-Maximilians-University of Munich, Munich, Germany; ${ }^{7}$ Department of \\ Orthopaedic Surgery, Orthopaedic Oncology, Ludwig-Maximilians-University of Munich, Munich, Germany; ${ }^{8}$ Department of \\ Radiotherapy and Radiation Oncology, Ludwig-Maximilians-University of Munich, Munich, Germany; ${ }^{9}$ Department of Clinical \\ Radiology, Ludwig-Maximilians-University of Munich, Munich, Germany; ${ }^{10}$ Department of Nuclear Medicine, Vienna General Hospital, \\ Vienna, Austria; and ${ }^{11}$ Department of Internal Medicine III, Ludwig-Maximilians-University of Munich, Munich, Germany
}

\begin{abstract}
We evaluated the prognostic accuracy of established PET and CT response criteria in patients with soft-tissue sarcoma (STS) after combined chemotherapy plus regional hyperthermia (RHT). Methods: Seventy-three patients underwent ${ }^{18} \mathrm{~F}-\mathrm{FDG}$ PET/CT before and after $2-4$ cycles of neoadjuvant chemotherapy with RHT for STS. Progressionfree survival (PFS) and time to local and distant progression were among other factors correlated with response according to PET Response Criteria in Solid Tumors (PERCIST 1.0) and Response Evaluation Criteria in Solid Tumors (RECIST 1.1). Results: Metabolic response by PERCIST $(n=44 / 73)$ was an independent predictor for PFS $(P=0.002$; hazard ratio $[\mathrm{HR}], 0.35$; 95\% confidence interval $[\mathrm{Cl}], 0.18-0.68$ ) and time to local or distant progression. Other independent predictors for PFS by multivariate analysis were adjuvant radiotherapy $(P=0.010$; HR, 0.39 ; $95 \% \mathrm{Cl}, 0.20-0.80)$ and a baseline tumor size less than $5.7 \mathrm{~cm}(P=$ 0.012 ; HR, 0.43; 95\% Cl, 0.22-0.83). Response by RECIST 1.1 was seen in a small group of patients $(n=22 / 73)$ and allowed prediction of PFS for patients with sarcoma outside the abdomen $(P=0.048$; HR, $0.13 ; 95 \% \mathrm{Cl}, 0.02-0.98)$. Conclusion: Metabolic response by ${ }^{18} \mathrm{~F}-\mathrm{FDG}$ PET predicts PFS and time to local and distant progression after 2-4 cycles of neoadjuvant chemotherapy plus RHT for STS.
\end{abstract}

Key Words: survival; soft-tissue sarcoma; PET; FDG; hyperthermia

J Nucl Med 2015; 56:530-537

DOI: 10.2967/jnumed.114.152462

$\mathbf{S}$

\footnotetext{
Received Dec. 4, 2014; revision accepted Jan. 29, 2015.

For correspondence or reprints contact: Wolfgang P. Fendler, Department

of Nuclear Medicine, Marchioninistrasse 15, 81377 Munich, Germany.

E-mail: wolfgang.fendler@med.uni-muenchen.de

Published online Feb. 26, 2015.

COPYRIGHT (c) 2015 by the Society of Nuclear Medicine and Molecular Imaging, Inc.
}

patients are diagnosed with aggressive high-grade histologic types at initial diagnosis (1). The therapy in these patients often entails a multimodal approach, which includes chemotherapy, radiotherapy, and resection. Regional hyperthermia (RHT) acts synergistically with chemotherapy and radiotherapy (2). In a previous prospective multicenter phase III trial with 341 patients with high-risk STS, there was an improved rate for treatment response, local progression-free survival (PFS), and disease-free survival on addition of RHT to standard neoadjuvant chemotherapy $(3,4)$. Despite an improved local effect, $19 \%$ of the patients in the RHT arm had local progression and $24 \%$ had distant progression at $2 \mathrm{y}$. As there are various local and systemic approaches to STS treatment, it is crucial to identify early those patients with high risk for relapse, to adapt optimally their treatment. However, histopathologic response is only available in a setting of resectable disease, and CT-based changes in tumor size by Response Evaluation Criteria in Solid Tumors (RECIST) are inaccurate for STS responsemonitoring (5-7). On the other hand, metabolic imaging with PET using the glucose analog ${ }^{18} \mathrm{~F}-\mathrm{FDG}$ has been effective for predicting tumor grade and outcome after chemotherapy based on early or late changes in tumor metabolism (6-11). However, the feasibility and prognostic value of metabolic response has not yet been investigated in a setting of combined chemotherapy plus RHT. We thus hypothesized that changes in tumor metabolism from baseline to intermediate ${ }^{18} \mathrm{~F}-\mathrm{FDG}$ PET/CT might predict local PFS and time to local or distant progression in patients with STS undergoing combined neoadjuvant chemotherapy plus RHT.

\section{MATERIALS AND METHODS}

\section{Patients}

Seventy-three consecutive patients who underwent ${ }^{18}$ F-FDG PET/ CT before and after neoadjuvant chemotherapy with RHT for STS between December 2008 and June 2013 were retrospectively selected from a local database including patients from a prospective observational study 
based on the international multicenter trial Combination Chemotherapy With or Without Hyperthermia Therapy in Treating Patients with Soft Tissue Sarcoma (ClinicalTrials.gov number NCT00003052) treatment protocol $(n=583)$ (3). Inclusion criteria were histologically proven STS as defined by the World Health Organization (12); neoadjuvant chemotherapy plus RHT for primary disease or recurrence; baseline ${ }^{18} \mathrm{~F}-\mathrm{FDG}$ PET/CT at a maximum of $30 \mathrm{~d}$ before the start of therapy; and follow-up PET/CT after the second, third, or fourth cycle of chemotherapy and no later than 4 mo after the start of therapy. Exclusion criteria were no detectable tumor on baseline PET/CT, as defined by a CT diameter of less than $1 \mathrm{~cm}$; tumor maximum standardized uptake value $\left(\mathrm{SUV}_{\max }\right)$ less than mean liver uptake; and blood glucose greater than $150 \mathrm{mg} / \mathrm{dL}$ at baseline or follow-up PET/CT. A flow diagram for selection of the study cohort is shown in Supplemental Figure 1 (supplemental materials are available at http://jnm.snmjournals.org). Our retrospective analysis of a prospective patient cohort was approved by the local ethics committee, and the requirement to obtain informed consent was waived.

\section{Neoadjuvant Therapy}

Sixty-six patients $(90 \%)$ received neoadjuvant chemotherapy with ifosfamide $\left(6-9 \mathrm{~g} / \mathrm{m}^{2}\right)$ and doxorubicin $\left(60 \mathrm{mg} / \mathrm{m}^{2}\right)$ (ifosfamide doxorubicin regimen [AI regimen]). In 7 patients, epirubicin $\left(90 \mathrm{mg} / \mathrm{m}^{2}\right)$ and dacarbazine $\left(250 \mathrm{mg} / \mathrm{m}^{2}\right)$ (epirubicin dacarbazine regimen [EDIC regimen]) or ifosfamide $\left(6 \mathrm{~g} / \mathrm{m}^{2}\right)$, carboplatin $\left(400 \mathrm{mg} / \mathrm{m}^{2}\right)$, and etoposide $\left(600 \mathrm{mg} / \mathrm{m}^{2}\right)$ (mini-ifosfamide carboplatin etoposide regimen [ICE regimen]) were chosen to adapt toxicity. The time interval between the cycles was $3 \mathrm{wk}$ for AI and EDIC and 4 wk for mini-ICE. RHT aiming for tumor temperatures of $42^{\circ} \mathrm{C} \pm 1{ }^{\circ} \mathrm{C}$ for $60 \pm 5$ min was given on days 1 and 4 of each chemotherapy cycle. Further treatment was determined by decision of a multidisciplinary tumor board. In the case of resectable disease, definite tumor excision was performed within 4-6 wk after induction therapy. Radiotherapy was performed 4-6 wk after surgery if indicated. A diagram of the study design is shown in Supplemental Figure 2.

\section{PET/CT Imaging}

Patients fasted at least $6 \mathrm{~h}$ to achieve a median blood glucose level of $93 \mathrm{mg} / \mathrm{dL}$ (interquartile range [IQR], $15 \mathrm{mg} / \mathrm{dL}$ ) at the time of ${ }^{18} \mathrm{~F}-$ FDG injection. Whole-body ${ }^{18} \mathrm{~F}-\mathrm{FDG}$ PET/CT images were acquired using a Biograph 64 TruePoint PET/CT scanner $(n=137 ; 94 \%)$ or a Discovery 690 scanner $(n=9 ; 6 \%$; GE Healthcare). Emission scans were initiated $90 \mathrm{~min}$ after almost simultaneous intravenous administration of $20 \mathrm{mg}$ of furosemide, $10 \mathrm{mg}$ of butylscopolamine, and ${ }^{18} \mathrm{~F}-\mathrm{FDG}$. Patients received a median body weight-adjusted dose of $254 \mathrm{MBq}$ (IQR, $32 \mathrm{MBq})$ of ${ }^{18} \mathrm{~F}-\mathrm{FDG}$. Of the 146 diagnostic CT scans (100-190 mAs, depending on the scanned organ region, $120 \mathrm{kV}), 136$ (93\%) were acquired with an intravenous injection of iodine-containing contrast agent (Ultravist 300 [Schering] or Imeron 300 [Bracco]; $2.5 \mathrm{~mL} / \mathrm{s}$ ) at a dose adjusted for body weight, and $10(7 \%)$ were acquired with no contrast agent. To allow valid pooling of the results, phantom studies based on the National Electrical Manufacturers Association NU2-2001 standard were conducted, and standardized uptake value (SUV) conversion factors were calculated (13-15).

\section{Response Assessment}

CT response was determined on target and nontarget lesions in accordance with RECIST 1.1 (16). PET response was determined by changes in peak SUV ( $\left.\mathrm{SUV}_{\text {peak }}\right)$ from baseline to follow-up in accordance with modified PET Response Criteria in Solid Tumors (PERCIST 1.0) (17). The following modifications were used: body weightadjusted SUV was calculated rather than lean body mass-adjusted SUL, and $\mathrm{SUV}_{\text {peak }}$ of a measurable target lesion was at least 2 SDs above the mean liver SUV, rather than 1.5-fold of the mean liver SUV plus 2 SDs. This less stringent cutoff was chosen because several studies reported low ${ }^{18} \mathrm{~F}-\mathrm{FDG}$ uptake in certain STS lesions, especially in the grade 1 and 2 categories $(11,18)$. Mean healthy liver SUV was defined as mean SUV computed in a $15 \mathrm{~cm}^{3}$ cubic volume of interest placed in the center of the right hepatic lobe. CT and PET responses were categorized separately as findings of progressive disease, stable disease, partial response, or complete response. For binary hazard analysis, any response was defined as the presence of partial response or complete response. No response was defined as the presence of stable disease or progressive disease. CT diameter and SUV measurements were performed by 2 interpreters together, each with more than $5 \mathrm{y}$ of experience in clinical and scientific interpretation of ${ }^{18} \mathrm{~F}-\mathrm{FDG}$ PET/CT scans for tumor staging using a 3-dimensional Volume Rendering Tool (Hybrid Viewer 3D for research; Hermes Medical Solutions).

\section{Histopathology}

Tumors were categorized in accordance with World Health Organization Classification of Tumors of Soft Tissue and Bone (2013) (12). Tumor grading was based on the 3-tier French Fédération Nationale des Centers de Lutte Contre le Cancer system (12). Histopathologic regression after chemotherapy was grouped by proportion of vital tumor in the resected tissue, in accordance with a scale proposed by SalzerKuntschik et al. for osteosarcoma (19). Grades 1-3 were defined as histopathologic response. Readings were performed by a soft-tissue and bone pathologist with more than $5 \mathrm{y}$ of experience, who was masked to PET and CT data.

\section{Outcome Assessment}

Clinical follow-up of patients was performed at least every 6 mo or at a shorter interval, as clinically indicated. Follow-up consisted of standard evaluations including clinical examination and imaging. The primary outcome was PFS as defined by the U.S. Food and Drug Administration (20). Secondary endpoints were time to local event $\left(\mathrm{TTE}_{\text {local }}\right)$, defined as the time from start of chemotherapy to objective local progression, and time to distant event $\left(\mathrm{TTE}_{\text {distant }}\right)$, defined as the time from start of chemotherapy to objective distant progression. Objective progression was suggested on CT, PET/CT, or MR imaging and was confirmed by informed decision of a multidisciplinary sarcoma board.

\section{Statistical Analysis}

Quantitative data are presented as number (percentage), median (with IQR), or mean \pm SD. The optimal cutoff for baseline tumor size and SUV was defined as the point on the receiver-operating-characteristic curve that was farthest from the line of equality (Youden index). Kaplan-Meier analysis of survival was performed on the basis of the interval between the start of chemotherapy until the last date of contact as censored observation, or until disease-related progression as the event of interest. Quantitative survival data are given as median (with IQR) in months. A log-rank test was used for statistical comparison of survival rates between independent subgroups. Univariate survival analysis was conducted by Cox proportional hazards regression. Parameters with a $P$ value of less than 0.20 in the univariate analysis were entered into a multivariate Cox regression model. In the multivariate analysis, we applied the Wald stepwise selection method with a $P$ value of 0.05 as entry probability and 0.10 as removal probability. Corresponding estimates for hazard ratio (HR) and $95 \%$ confidence interval (CI) were calculated. Significance was set at a $P$ value of less than 0.05. The SPSS software package (version 15.0; SPSS, Inc.) was used for all statistical analysis.

\section{RESULTS}

\section{Characteristics of Study Cohort and Survival}

Patient characteristics are presented in Table 1 . Most of the 73 STS cases were primary high-grade tumors $(55 ; 75 \%)$ frequently of retroperitoneal location $(36 ; 49 \%)$. The median PFS was 15.3 
TABLE 1

Characteristics of Study Cohort

\begin{tabular}{|c|c|}
\hline Patient characteristic $(n=73)$ & Median or absolute number* \\
\hline Age $(y)$ & $52(\mathrm{IQR}, 24)$ \\
\hline \multicolumn{2}{|l|}{ Sex } \\
\hline Female & $37(51 \%)$ \\
\hline Male & $36(49 \%)$ \\
\hline \multicolumn{2}{|l|}{ Site } \\
\hline Head and neck & $3(4 \%)$ \\
\hline Chest and trunk & $7(10 \%)$ \\
\hline Abdomen and retroperitoneum & $36(49 \%)$ \\
\hline Extremities & 27 (37\%) \\
\hline \multicolumn{2}{|l|}{ Histology } \\
\hline Angiosarcoma & $6(8 \%)$ \\
\hline Leiomyosarcoma & $13(18 \%)$ \\
\hline Liposarcoma & $13(18 \%)$ \\
\hline MPNST & $6(8 \%)$ \\
\hline Synovial sarcoma & $4(5 \%)$ \\
\hline Pleomorphic undifferentiated sarcoma & $15(21 \%)$ \\
\hline Other & $16(22 \%)$ \\
\hline \multicolumn{2}{|l|}{ FNCLCC grade } \\
\hline 1 & $1(1 \%)$ \\
\hline 2 & $22(30 \%)$ \\
\hline 3 & $50(69 \%)$ \\
\hline \multicolumn{2}{|l|}{ Stage } \\
\hline Recurrent tumor & $18(25 \%)$ \\
\hline Primary tumor & 55 (75\%) \\
\hline \multicolumn{2}{|l|}{ Radiotherapy } \\
\hline Yes & $30(41 \%)$ \\
\hline No & $43(59 \%)$ \\
\hline \multicolumn{2}{|l|}{ Surgery } \\
\hline After chemotherapy & $34(47 \%)$ \\
\hline Before chemotherapy & $27(37 \%)$ \\
\hline No surgery & $12(16 \%)$ \\
\hline \multicolumn{2}{|l|}{ Chemotherapy } \\
\hline Al & $66(90 \%)$ \\
\hline EDIC & $3(4 \%)$ \\
\hline Mini-ICE & $4(6 \%)$ \\
\hline \multicolumn{2}{|l|}{ Progression (events) } \\
\hline Local & $27(37 \%)$ \\
\hline Distant & $24(33 \%)$ \\
\hline Local plus distant & $37(51 \%)$ \\
\hline Deaths & $19(26 \%)$ \\
\hline Time to event overall (mo) & $15.3(\mathrm{IQR}, 17.7)$ \\
\hline Overall survival (mo) & $22.2($ IQR, 17.3) \\
\hline Time of follow-up (mo) & $22.2(\mathrm{IQR}, 17.3)$ \\
\hline No. of therapy courses between PET scans & $3(\mathrm{IQR}, 2)$ \\
\hline No. of therapy courses from second PET to surgery & $1(\mathrm{IQR}, 2)$ \\
\hline
\end{tabular}

${ }^{*}$ Data are median, with IQR in parentheses, or absolute number (with \% in parentheses).

MPNST = malignant peripheral nerve sheath tumor; FNCLCC $=$ French Fédération Nationale des Centers de Lutte Contre le Cancer. 
(IQR, 17.7) mo. Follow-up PET/CT was performed in 24 of $73(33 \%)$ patients after 2 cycles, in 17 patients $(23 \%)$ after 3 cycles, and in $32(44 \%)$ patients after 4 cycles of chemotherapy.

\section{PERCIST and RECIST Response}

The mean tumoral $\mathrm{SUV}_{\text {peak }}$ was $9.9 \pm 9.1$ (range, 2.8-62.8) at baseline and $6.3 \pm 8.4$ (range, 0.5-58.9) at follow-up. The mean baseline to follow-up change of $\mathrm{SUV}_{\text {peak }}$ was $-33 \% \pm 48 \%$ (range, $-94 \%$ to $202 \%$ ). In 8 of 73 patients $(11 \%)$, baseline to follow-up $\mathrm{SUV}_{\text {peak }}$ increased by a mean of $66 \% \pm 77 \%$ (range, $11 \%-202 \%)$. Sixteen of 73 patients (22\%) had complete response, $28(38 \%)$ had partial response, $25(34 \%)$ had stable disease, and 4 (5\%) had progressive disease according to PERCIST. The mean baseline to follow-up change of $\mathrm{SUV}_{\text {peak }}$ was $-35 \% \pm 28 \%$ (range, $-81 \%$ to $43 \%$ ) after 2 cycles, $-36 \% \pm 58 \%$ (range, $-94 \%$ to $172 \%$ ) after 3 cycles, and $-30 \% \pm 55 \%$ (range, $-88 \%$ to $202 \%$ ) after 4 cycles of chemotherapy.

The mean largest tumor diameter was $7.3 \pm 5.5 \mathrm{~cm}$ at baseline and $6.2 \pm 5.6 \mathrm{~cm}$ at follow-up. The mean baseline to follow-up change in the sum of the largest tumor diameters was $-24 \% \pm$ $32 \%$. In 12 of the 73 patients (16\%), baseline to follow-up diameters increased by a mean of $15 \% \pm 11 \%$. In 3 patients (4\%), new lesions appeared on follow-up CT. Two patients (3\%) had complete response, $20(27 \%)$ had partial response, $46(63 \%)$ had stable disease, and $5(7 \%)$ had progressive disease according to RECIST.

\section{Histopathologic Response}

Thirty-four of 73 (47\%) patients had surgery after induction chemotherapy. The median number of chemotherapy cycles between follow-up PET/CT and surgery was 1 (IQR, 2). The mean proportion of vital tumor tissue after chemotherapy was $37 \% \pm 34 \%$, and mean Salzer-Kuntschik grade was $3.5 \pm 1.7$. Thirteen patients (38\%) had less than $10 \%$ vital tumor tissue on histopathologic analysis (Salzer-Kuntschik grade 1-3) and were therefore classified as histopathologic responders.

\section{Survival Analysis}

Chemotherapy with RHT acts systemically with localized boosting by application of heat. To depict local and systemic effects, the response to therapy was expressed by 3 different outcome parameters - that is, the primary outcome, PFS, and 2 secondary endpoints, $\mathrm{TTE}_{\mathrm{local}}$ and $\mathrm{TTE}_{\text {distant }}$. Univariate analysis was conducted for PERCIST, RECIST, baseline size and $\mathrm{SUV}_{\text {peak }}$, histopathologic response, and characteristics of the patient cohort that had already been connected to patient outcome in a previous study (Tables 2 and 3) (21). Figures 1 and 2 show the corresponding Kaplan-Meier survival curves.

Both RECIST and PERCIST responses were significantly associated with PFS (Table 2). Patients with any response according to RECIST ( $n=22$ ) had a 2.7 -fold decrease in risk for progression or death $(P=0.016$; HR, $0.37 ; 95 \% \mathrm{CI}, 0.16-0.83)$; any response according to PERCIST $(n=44)$ was associated with a 3.7-fold decreased risk $(P<0.001$; HR, 0.27; 95\% CI, 0.14$0.51)$. The median PFS in patients with any response $(n=44)$ versus no response $(n=29)$ according to PERCIST was 16.9 (IQR, 21.6) versus 9.7 (IQR, 10.0) mo. The median PFS in patients with any response $(n=22)$ versus no response $(n=$ 51) according to RECIST was 19.1 (IQR, 23.9) versus 15.0 (IQR, 15.8) mo. Patients with no response according to RECIST were further divided by PERCIST into patients with any response ( $n=28$; median PFS, 24 [IQR, 19] mo) or no response $(n=23$; median PFS, 10 [IQR, 13] mo). PFS was significantly different between these subgroups according to the log-rank test $(P=$ 0.017 , Fig. 3). Figure 4 shows images from a representative patient with stable disease according to RECIST and PR according to PERCIST. In 16 patients, both RECIST and PERCIST predicted therapy response. The median PFS of these patients was 27.8 (IQR, 19.9) mo. In 23 patients, neither criterion predicted a response; the median PFS for this group was 9.7 (IQR, 11.9) mo.

TABLE 2

Univariate Cox Regression Analysis for PFS

\begin{tabular}{|c|c|c|c|c|c|}
\hline \multirow[b]{2}{*}{ Variable } & \multirow[b]{2}{*}{ Subcategory } & \multirow[b]{2}{*}{$n$} & \multicolumn{3}{|c|}{ PFS } \\
\hline & & & $P$ & $\mathrm{HR}$ & $95 \% \mathrm{Cl}$ \\
\hline Age (y) & $\geq 50$ & 43 & 0.948 & 0.98 & $0.52-1.84$ \\
\hline Recurrence & Yes & 18 & 0.471 & 1.28 & $0.65-2.51$ \\
\hline FNCLCC grade & 3 & 50 & 0.836 & 0.94 & $0.50-1.77$ \\
\hline Resection margin & Positive & 36 & 0.974 & 1.01 & $0.49-2.09$ \\
\hline Radiotherapy & Yes* & $30^{*}$ & $0.004^{\star \dagger}$ & $0.37^{\star}$ & $0.19-0.72^{*}$ \\
\hline Size $(\mathrm{cm})$ & $<5.7^{\star}$ & $36^{\star}$ & $0.008^{\star}+$ & $0.42^{\star}$ & $0.22-0.80^{\star}$ \\
\hline SUV $_{\text {peak }}$ & $<10.7^{\star}$ & $48^{*}$ & $0.028^{\star \dagger}$ & $0.50^{\star}$ & $0.27-0.93^{\star}$ \\
\hline RECIST & $\begin{array}{l}\text { Any response (complete response } \\
\text { or partial response)* }\end{array}$ & $22^{*}$ & $0.016^{\star \dagger}$ & $0.37^{\star}$ & $0.16-0.83^{\star}$ \\
\hline PERCIST & $\begin{array}{l}\text { Any response (complete response } \\
\text { or partial response)* }\end{array}$ & $44^{*}$ & $<0.001^{\star}+$ & $0.27^{\star}$ & $0.14-0.51^{*}$ \\
\hline Histopathologic response & SK 1-3 & 13 & 0.180 & 0.45 & $0.14-1.45$ \\
\hline \multicolumn{6}{|l|}{$\begin{array}{l}{ }^{\star} \text { Statistical significance. } \\
{ }^{\dagger} P<0.05\end{array}$} \\
\hline
\end{tabular}


TABLE 3

Univariate Cox Regression Analysis for $T \mathrm{TE}_{\text {local }}$ or $T T E_{\text {distant }}$

\begin{tabular}{|c|c|c|c|c|c|c|c|c|}
\hline \multirow[b]{2}{*}{ Variable } & \multirow[b]{2}{*}{ Subcategory } & \multirow[b]{2}{*}{$n$} & \multicolumn{3}{|c|}{$\mathrm{TTE}_{\text {local }}$} & \multicolumn{3}{|c|}{$\mathrm{TTE}_{\text {distant }}$} \\
\hline & & & $P$ & $\mathrm{HR}$ & $95 \% \mathrm{Cl}$ & $P$ & $\mathrm{HR}$ & $95 \% \mathrm{Cl}$ \\
\hline Age (y) & $\geq 50$ & 43 & 0.716 & 1.16 & $0.53-2.53$ & 0.522 & 0.77 & $0.34-1.72$ \\
\hline Recurrence & Yes & 18 & 0.639 & 1.22 & $0.53-2.79$ & 0.281 & 1.60 & $0.68-3.73$ \\
\hline FNCLCC grade & 3 & 50 & 0.517 & 1.32 & $0.58-3.01$ & 0.760 & 0.88 & $0.39-2.01$ \\
\hline Resection margin & Positive & 36 & 0.478 & 1.39 & $0.56-3.45$ & 0.476 & 0.73 & $0.30-1.76$ \\
\hline Radiotherapy & Yes $^{*}$ & $30^{*}$ & $0.001^{\star *}$ & $0.18^{\star}$ & $0.06-0.51^{*}$ & 0.222 & 0.59 & $0.26-1.37$ \\
\hline Size $(\mathrm{cm})$ & $<5.7^{\star}$ & $36^{\star}$ & $0.020^{\star \dagger}$ & $0.38^{*}$ & $0.17-0.86^{*}$ & 0.204 & 0.59 & $0.26-1.33$ \\
\hline SUV $_{\text {peak }}$ & $<10.7^{\star}$ & $48^{*}$ & $0.038^{\star \dagger}$ & $0.45^{\star}$ & $0.21-0.96^{*}$ & 0.969 & 0.98 & $0.41-2.38$ \\
\hline RECIST & $\begin{array}{l}\text { Any response (complete response } \\
\text { or partial response) }\end{array}$ & $22^{*}$ & 0.115 & 0.46 & $0.17-1.21$ & $0.036^{\star} \dagger$ & $0.27^{\star}$ & $0.08-0.92^{\star}$ \\
\hline PERCIST & $\begin{array}{l}\text { Any response (complete response } \\
\text { or partial response) }\end{array}$ & $44^{*}$ & $0.018^{\star \dagger}$ & $0.34^{*}$ & $0.18-0.86^{\star}$ & $0.004^{\star \dagger}$ & $0.29^{*}$ & $0.13-0.68^{*}$ \\
\hline Histopathologic response & SK 1-3 & 13 & 0.211 & 0.37 & $0.08-1.77$ & 0.724 & 0.77 & $0.18-3.25$ \\
\hline \multicolumn{9}{|l|}{$\begin{array}{l}{ }^{\star} \text { Statistical significance. } \\
{ }^{\dagger} P<0.05 . \\
\text { FNCLCC }=\text { French Fédéra }\end{array}$} \\
\hline
\end{tabular}

For subgroup analysis, the study cohort was divided by tumor location into those patients with retroperitoneal sarcoma $(n=36$; $49 \%$ ) versus patients with a sarcoma at any other location (i.e., chest, neck, head, or extremities; $n=37 ; 51 \%$ ). In the subgroup of patients with retroperitoneal sarcoma, PERCIST significantly predicted PFS $(P=0.009 ; \mathrm{HR}, 0.33 ; 95 \% \mathrm{CI}, 0.14-0.75)$, whereas RECIST did not indicate primary outcome $(P=0.331)$. For the 37 patients with sarcoma of the chest, neck, head, or extremities, both parameters correlated significantly with PFS $(P=0.048$ and 0.010 , respectively). Results for univariate analysis in these 2 groups are given in Supplemental Table 1. Furthermore, patients with primary highgrade tumors $(n=55)$ were analyzed separately. In this subgroup, both PERCIST $(P=0.001$; HR, 0.26 ; 95\% CI, 0.12-0.56) and RECIST $(P=0.044$; HR, 0.34; 95\% CI, 0.12-0.97) responses significantly predicted PFS (Fig. 5).

Type of neoadjuvant treatment (chemotherapy alone vs. chemotherapy plus radiotherapy), baseline tumor size, and $\mathrm{SUV}_{\text {peak }}$ were significantly associated with PFS for the entire cohort, whereas his- topathologic response, choice of chemotherapy regimen (data not shown), and the remaining patient characteristics (age; stage of occurrence, i.e., primary or recurrence; margin positivity) did not predict primary or secondary outcomes. On multivariate analysis, additional radiotherapy $(P=0.010$; HR, 0.39 ; 95\% CI, 0.20 $0.80)$, baseline size less than $5.7 \mathrm{~cm}(P=0.012$; HR, $0.43 ; 95 \%$ CI, 0.22-0.83), and PERCIST response $(P=0.002$; HR, 0.35; 95\% CI, 0.18-0.68) were the only independent predictors of PFS.

\section{TTE $_{\text {local }}$ or TTE distant $_{\text {. }}$}

Both RECIST and PERCIST predicted TTE $_{\text {distant, }}$, with significant reductions in hazard (12.2- and 8.0-fold; $P=0.036$ and 0.004 , respectively), although only PERCIST correlated with local progression $(P=0.018$; HR, $0.40 ; 95 \%$ CI, 0.18-0.86). Type of neoadjuvant treatment (chemotherapy alone vs. chemotherapy plus radiotherapy), baseline size, and $\mathrm{SUV}_{\text {peak }}$ were significantly associated with $\mathrm{TTE}_{\text {local }}(P<0.05$ each $)$, whereas they did not predict distant events.

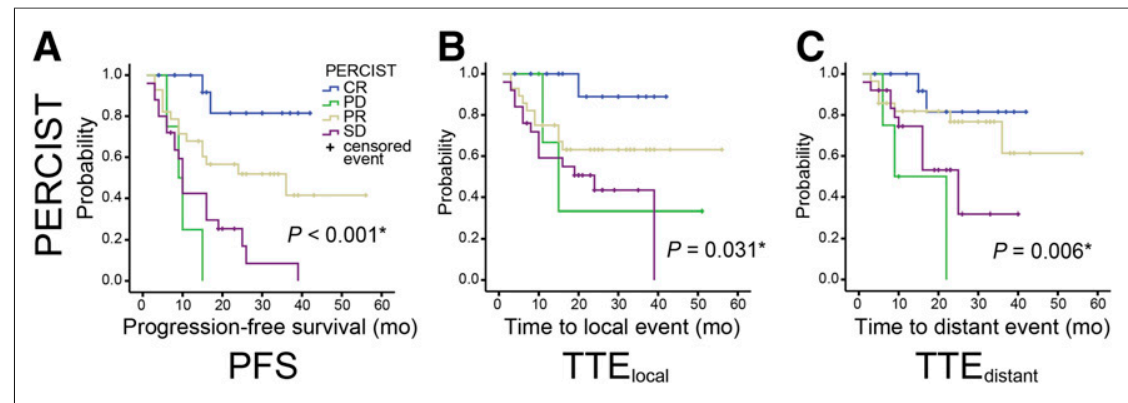

FIGURE 1. Kaplan-Meier survival for $P F S(A), T T E_{\text {local }}(B)$, and $T T E_{\text {distant }}(C)$ in mo as determined by PERCIST $(n=73)$. CR $=$ complete response; $\mathrm{PD}=$ progressive disease; $\mathrm{PR}=$ partial response; $\mathrm{SD}=$ stable disease. ${ }^{*} P<0.05$.

\section{DISCUSSION}

Our study shows that treatment response can be predicted by ${ }^{18} \mathrm{~F}-\mathrm{FDG}$ PET/CT after 2-4 cycles of combined neoadjuvant chemotherapy plus RHT in a series of 73 patients with locally advanced high-grade STS. Other research groups have likewise reported a significant correlation between PET response and survival or histopathologic response in patients with STS after 1 cycle $(5,7)$, after 2 cycles (22), or after completion (6) of neoadjuvant chemotherapy, albeit without concomitant RHT, which was integral to the present study. Our study indicates that ${ }^{18}$ F-FDG PET/CT has considerable 


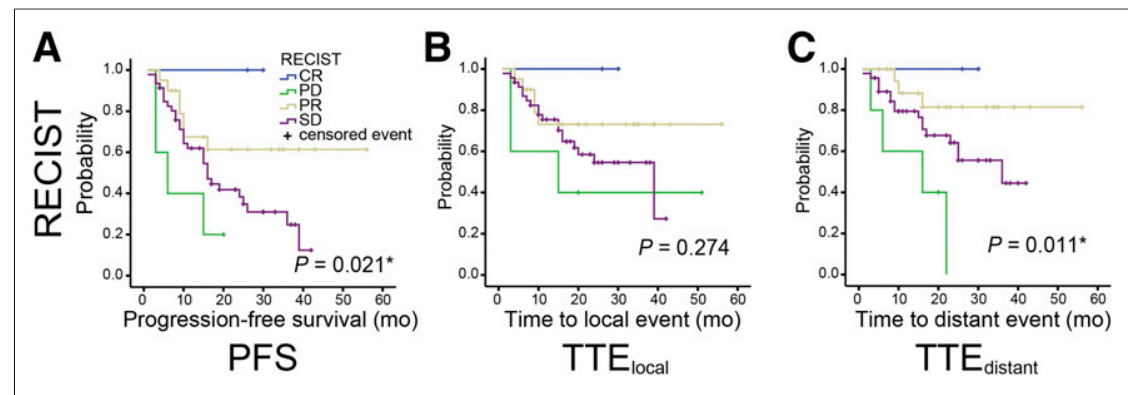

FIGURE 2. Kaplan-Meier survival for $P F S(A), T T E_{\text {local }}(B)$, and $T T E_{\text {distant }}(C)$ in mo as determined by RECIST $(n=73)$. $\mathrm{CR}=$ complete response; $\mathrm{PD}=$ progressive disease; $\mathrm{PR}=$ partial response; $\mathrm{SD}=$ stable disease. ${ }^{*} P<0.05$.

potential to guide treatment after a combined chemotherapy plus RHT, in that adjuvant chemotherapy or radiation therapy can be adapted in nonresponding patients. Risk-adapted therapy might ultimately reduce treatment-associated toxicity and costs in patients receiving a combined treatment.

Metabolic response by PERCIST was an independent predictor of PFS. PERCIST response was associated with the strongest reduction in risk (about 3.7-fold decreased hazard) in all patients and in patients with high-grade tumors at primary diagnosis. Response according to PERCIST was furthermore seen in the largest group of patients $(n=44,60 \%)$. On the other hand, change in tumor size did not independently predict PFS. A large group of patients with no significant change in tumor size after chemotherapy showed considerable variation in PFS and could further be differentiated on the basis of PET criteria into subgroups with good versus poor outcome. Several previous studies on STS have similarly shown that changes in tumor size are only poorly correlated with survival or histopathologic response after chemotherapy, especially in the early follow-up period $(6,7)$. We concluded that changes in tumor size tend to occur later

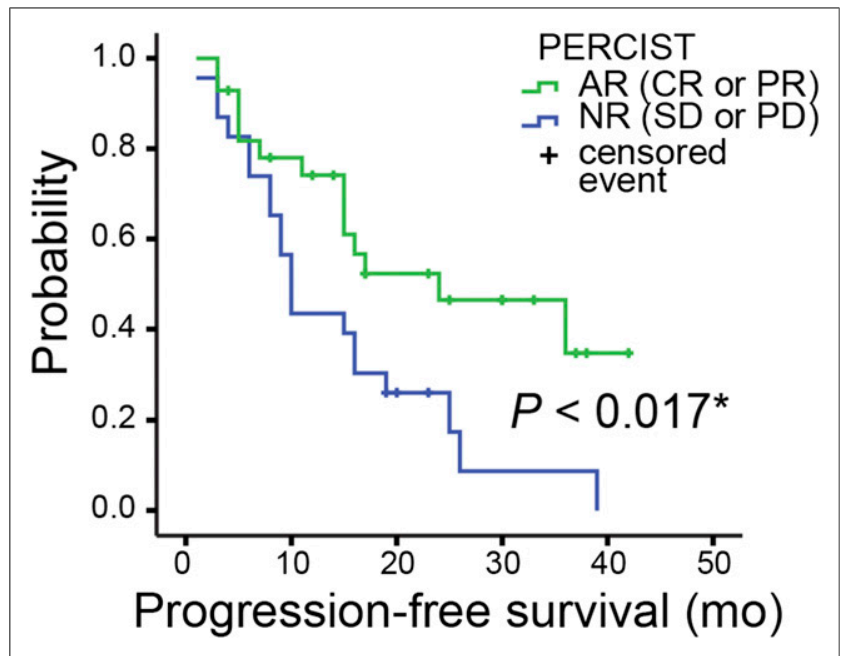

FIGURE 3. Kaplan-Meier survival curves for PFS in patients with no response according to RECIST ( $n=51$ ), shown separately for subgroups with any response (AR) according to PERCIST ( $n=28$, green curve) and no response (NR) according to PERCIST ( $n=23$, blue curve). Survival is given in mo. Patients with PERCIST AR had significantly longer PFS than PERCIST NR $(P=0.017)$. $C R=$ complete response; $P D=$ progressive disease; $\mathrm{PR}=$ partial response; $\mathrm{SD}=$ stable disease. ${ }^{\star} P<0.05$. during therapy, whereas a decrease in tumor metabolic rate is an early hallmark of eventual treatment response. This difference was even more evident for cases of local recurrence; PERCIST predicted local recurrence with a similar reduction in risk, whereas change in tumor size did not correlate with time to local progression.

There have been concerns that RHTinduced changes in the tumor microenvironment might influence glycolysis and ${ }^{18} \mathrm{~F}-\mathrm{FDG}$ uptake. Indeed, persistent increase in lymphocyte and macrophage recruitment has been described after application of local heat in preclinical tumor models (23), which might impart physiologic ${ }^{18}$ F-FDG uptake. The extent of tumor necrosis after chemotherapy may thus be underestimated, given the additional ${ }^{18} \mathrm{~F}-\mathrm{FDG}$ uptake arising from leukocyte infiltrates. Nonetheless, we found a significant reduction in ${ }^{18} \mathrm{~F}-\mathrm{FDG}$ uptake after chemotherapy, which argues against this putative consequence of RHT in our patient population. We concluded that the application of local heat does not induce a notably persistent effect on tumor glycolysis that might potentially interfere with the accuracy of ${ }^{18} \mathrm{~F}-\mathrm{FDG}$ PET. Thus, changes in tumor metabolism could predict local and overall progression more accurately than the other imaging parameters in our study.

Both RECIST and PERCIST predicted distant progression with similar reduction in risk, albeit in a considerably larger group of PERCIST responders than RECIST responders (44 vs. 22 patients). In our study, metabolic response by PERCIST was significantly associated with improved PFS in patients with retroperitoneal sarcoma, whereas change in tumor size did not predict primary outcome. In most cases, sarcomas of the retroperitoneum are diagnosed late, at times when tumors had already

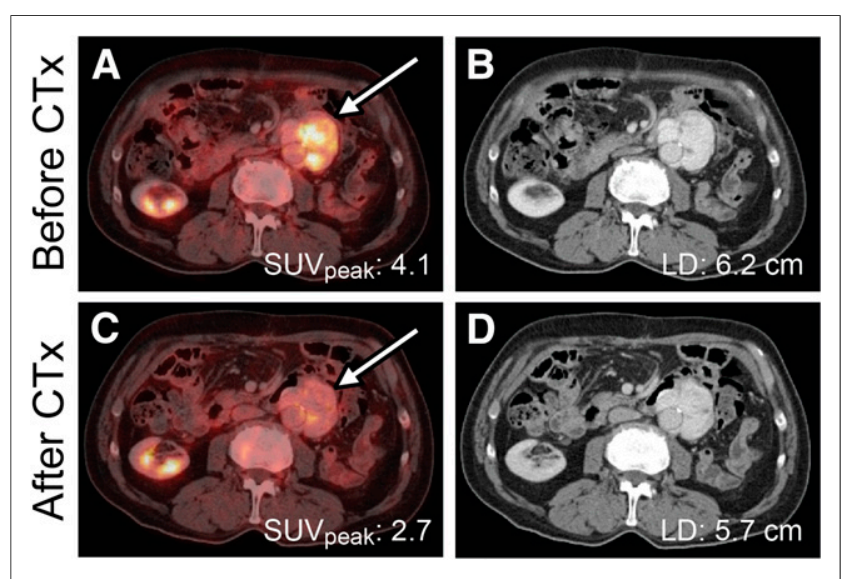

FIGURE 4. A 78-y-old male patient with liposarcoma encasing abdominal aorta (arrow). Axial CT and fused ${ }^{18} \mathrm{~F}-\mathrm{FDG}$ PET/CT images are shown at baseline (A and $B$ ) and after 2 cycles of neoadjuvant chemotherapy (CTx) (C and D). Largest CT diameter (LD) was $6.2 \mathrm{~cm}$ at baseline and $5.7 \mathrm{~cm}$ at follow-up (8\% decrease; SD according to RECIST). SUV peak $_{\text {was }} 4.1$ at baseline and 2.7 at follow-up (34\% decrease; PR according to PERCIST). Patient had PFS of $24 \mathrm{mo}$, compared with median PFS of 15.3 (IQR, 17.7) mo for entire study cohort. 


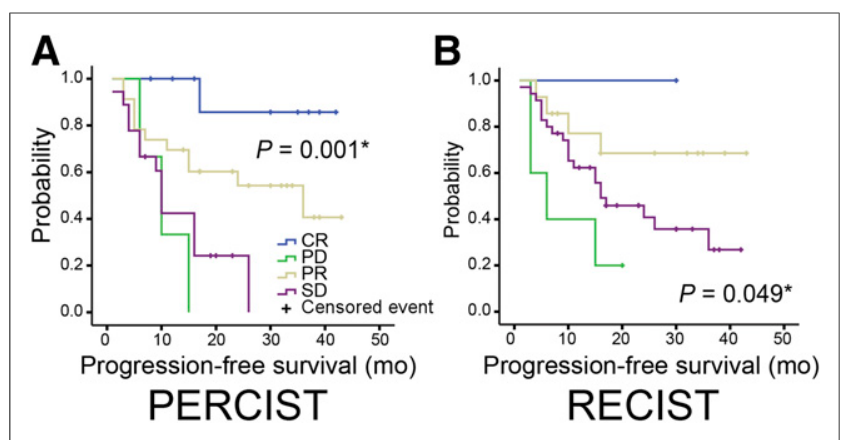

FIGURE 5. Kaplan-Meier survival curves for PFS in patients with primary high-grade tumors $(n=55)$ in mo grouped by PERCIST (A) and RECIST (B). $\mathrm{CR}=$ complete response; $\mathrm{PD}=$ progressive disease; $\mathrm{PR}=$ partial response; $\mathrm{SD}=$ stable disease. ${ }^{\star} P<0.05$.

become symptomatic due to their extensive growth and spaceoccupying effects. At such advanced stages, the volume of large masses is only poorly described by the index of largest diameter, and changes in tumor volume can be limited, given the considerable amounts of necrosis, hemorrhage, and edema in large lesions.

Both baseline size and $\mathrm{SUV}_{\text {peak }}$ predicted PFS and local recurrence with high accuracy. However, baseline cutoff values for responders were determined by receiver-operating-characteristic analysis and thus optimized for our particular patient cohort. This optimization imparts an advantage in prognostic accuracy for both baseline parameters relative to RECIST or PERCIST alone. Several previous reports suggest cutoff values other than those defined by RECIST or PERCIST, for example, an SUV cutoff greater than $30 \%$ for metabolic responders $(7,24)$. Because we intended in this study to test for the applicability of well-established systems, we did not alter RECIST or PERCIST cutoff values. The addition of radiotherapy was significantly associated with a decreased risk for local recurrence and PFS in our patient cohort, most likely due to an improved rate of local control, which has also been unequivocally found by a previous review of 39 studies on radiotherapy for STS (25).

Histopathologic response and inadequate resection did not emerge as significant predictors for PFS in our study, although both of these factors have been prognostic in previous studies on large cohorts using overall survival for patient outcome $(21,26)$. The discrepancy may in part be explained by the different endpoints, but also by the smaller group of patients receiving surgery after chemotherapy in our study. Herrmann et al. similarly found no significant difference in survival for histopathologic responders and noted that a cohort larger than 200 patients would be needed to detect a possible difference (7). Though patients were recruited from a prospective database, generalization of our findings may be limited by the retrospective study design and by the use of PFS and TTE instead of overall survival to assess treatment response.

\section{CONCLUSION}

Metabolic response by PERCIST after 2-4 cycles of chemotherapy in combination with RHT is an independent predictor for PFS as well as for local and distant progression in STS patients. Response by RECIST was seen in a small group of patients and allowed prediction of PFS for nonretroperitoneal STS. We did not see evidence for persistent effects of RHT on tumor glycolysis and thus no significant interference with prognostic accuracy of ${ }^{18}$ F-FDG PET after combined treatment. Our findings support carrying out prospective trials to examine whether metabolic response can be used to guide treatment decisions in STS to reduce treatment-related toxicity. This conclusion might generalize to other tumor entities for which combined chemotherapy plus RHT is indicated.

\section{DISCLOSURE}

The costs of publication of this article were defrayed in part by the payment of page charges. Therefore, and solely to indicate this fact, this article is hereby marked "advertisement" in accordance with 18 USC section 1734 . No potential conflict of interest relevant to this article was reported.

\section{ACKNOWLEDGMENT}

We acknowledge manuscript revision by Dr. Paul Cumming.

\section{REFERENCES}

1. Guillou L, Coindre JM, Bonichon F, et al. Comparative study of the National Cancer Institute and French Federation of Cancer Centers Sarcoma Group grading systems in a population of 410 adult patients with soft tissue sarcoma. J Clin Oncol. 1997;15:350-362.

2. Wust P, Hildebrandt B, Sreenivasa G, et al. Hyperthermia in combined treatment of cancer. Lancet Oncol. 2002;3:487-497.

3. Issels RD, Lindner LH, Verweij J, et al. Neo-adjuvant chemotherapy alone or with regional hyperthermia for localised high-risk soft-tissue sarcoma: a randomised phase 3 multicentre study. Lancet Oncol. 2010;11:561-570.

4. Angele MK, Albertsmeier M, Prix NJ, et al. Effectiveness of regional hyperthermia with chemotherapy for high-risk retroperitoneal and abdominal soft-tissue sarcoma after complete surgical resection: a subgroup analysis of a randomized phase-III multicenter study. Ann Surg. 2014;260:749-754.

5. Benz MR, Czernin J, Allen-Auerbach MS, et al. FDG-PET/CT imaging predicts histopathologic treatment responses after the initial cycle of neoadjuvant chemotherapy in high-grade soft-tissue sarcomas. Clin Cancer Res. 2009; 15:2856-2863.

6. Evilevitch V, Weber WA, Tap WD, et al. Reduction of glucose metabolic activity is more accurate than change in size at predicting histopathologic response to neoadjuvant therapy in high-grade soft-tissue sarcomas. Clin Cancer Res. 2008; 14:715-720.

7. Herrmann K, Benz MR, Czernin J, et al. ${ }^{18}$ F-FDG-PET/CT imaging as an early survival predictor in patients with primary high-grade soft tissue sarcomas undergoing neoadjuvant therapy. Clin Cancer Res. 2012;18:20242031.

8. Folpe AL, Lyles RH, Sprouse JT, Conrad EU, 3rd, Eary JF. (F-18) fluorodeoxyglucose positron emission tomography as a predictor of pathologic grade and other prognostic variables in bone and soft tissue sarcoma. Clin Cancer Res. 2000;6:1279-1287.

9. Benz MR, Allen-Auerbach MS, Eilber FC, et al. Combined assessment of metabolic and volumetric changes for assessment of tumor response in patients with soft-tissue sarcomas. J Nucl Med. 2008;49:1579-1584.

10. Eugene T, Corradini N, Carlier T, Dupas B, Leux C, Bodet-Milin C. ${ }^{18} \mathrm{~F}-$ FDG-PET/CT in initial staging and assessment of early response to chemotherapy of pediatric rhabdomyosarcomas. Nucl Med Commun. 2012;33: 1089-1095.

11. Benz MR, Dry SM, Eilber FC, et al. Correlation between glycolytic phenotype and tumor grade in soft-tissue sarcomas by ${ }^{18}$ F-FDG PET. J Nucl Med. 2010; 51:1174-1181.

12. International Agency for Research on Cancer (IARC). WHO Classification of Tumours of Soft Tissue and Bone (IARC WHO Classification of Tumours). 4th ed. Lyon, France: World Health Organization; 2013.

13. National Electrical Manufacturers Association (NEMA). NEMA NU 2: Performance Measurements of Positron Emission Tomographs. Rosslyn, VA: National Electrical Manufacturers Association; 2001. 
14. Haug AR, Tiega Donfack BP, Trumm C, et al. ${ }^{18} \mathrm{~F}-\mathrm{FDG}$ PET/CT predicts survival after radioembolization of hepatic metastases from breast cancer. $\mathrm{J} \mathrm{Nucl}$ Med. 2012;53:371-377.

15. Boellaard R. EANM Research Ltd (EARL). New EANM FDG PET/CT accreditation specifications for SUV recovery coefficients. EARL website. http://earl. eanm.org/cms/website.php?id=/en/projects/fdg_pet_ct_accreditation/accreditation_ specifications.htm. Accessed February 26, 2015.

16. Eisenhauer EA, Therasse P, Bogaerts J, et al. New response evaluation criteria in solid tumours: revised RECIST guideline (version 1.1). Eur J Cancer. 2009;45:228-247.

17. Wahl RL, Jacene H, Kasamon Y, Lodge MA. From RECIST to PERCIST: evolving considerations for PET response criteria in solid tumors. J Nucl Med. 2009;50(suppl 1):122S-150S.

18. Rakheja R, Makis W, Skamene S, et al. Correlating metabolic activity on ${ }^{18}$ F-FDG PET/CT with histopathologic characteristics of osseous and softtissue sarcomas: a retrospective review of 136 patients. AJR. 2012;198: 1409-1416.

19. Salzer-Kuntschik M, Delling G, Beron G, Sigmund R. Morphological grades of regression in osteosarcoma after polychemotherapy: study COSS 80. J Cancer Res Clin Oncol. 1983;106(suppl):21-24.
20. U.S. Food and Drug Administration. Guidance for Industry: Clinical Trial Endpoints for the Approval of Cancer Drugs and Biologics. Rockville, MD: U.S. Department of Health and Human Services; 2007.

21. Gaynor JJ, Tan CC, Casper ES, et al. Refinement of clinicopathologic staging for localized soft tissue sarcoma of the extremity: a study of 423 adults. J Clin Oncol. 1992;10:1317-1329.

22. Dimitrakopoulou-Strauss A, Strauss LG, Egerer G, et al. Impact of dynamic ${ }^{18} \mathrm{~F}-$ FDG PET on the early prediction of therapy outcome in patients with high-risk soft-tissue sarcomas after neoadjuvant chemotherapy: a feasibility study. $\mathrm{J} \mathrm{Nucl}$ Med. 2010;51:551-558.

23. Burd R, Dziedzic TS, Xu Y, Caligiuri MA, Subjeck JR, Repasky EA. Tumor cell apoptosis, lymphocyte recruitment and tumor vascular changes are induced by low temperature, long duration (fever-like) whole body hyperthermia. J Cell Physiol. 1998;177:137-147.

24. Schuetze SM, Rubin BP, Vernon C, et al. Use of positron emission tomography in localized extremity soft tissue sarcoma treated with neoadjuvant chemotherapy. Cancer. 2005;103:339-348.

25. Strander H, Turesson I, Cavallin-Stahl E. A systematic overview of radiation therapy effects in soft tissue sarcomas. Acta Oncol. 2003;42:516-531.

26. Collin C, Godbold J, Hajdu S, Brennan M. Localized extremity soft tissue sarcoma: an analysis of factors affecting survival. J Clin Oncol. 1987;5:601-612. 